\title{
Exploring Uncertainty Perception as a Driver of Design Activity
}

\author{
Cash, Philip; Kreye, Melanie
}

Published in:

Design Studies

Link to article, DOI:

10.1016/j.destud.2017.10.004

Publication date:

2018

Document Version

Peer reviewed version

Link back to DTU Orbit

Citation (APA):

Cash, P., \& Kreye, M. (2018). Exploring Uncertainty Perception as a Driver of Design Activity. Design Studies, 54, 50-79. https://doi.org/10.1016/j.destud.2017.10.004

\section{General rights}

Copyright and moral rights for the publications made accessible in the public portal are retained by the authors and/or other copyright owners and it is a condition of accessing publications that users recognise and abide by the legal requirements associated with these rights.

- Users may download and print one copy of any publication from the public portal for the purpose of private study or research.

- You may not further distribute the material or use it for any profit-making activity or commercial gain

- You may freely distribute the URL identifying the publication in the public portal

If you believe that this document breaches copyright please contact us providing details, and we will remove access to the work immediately and investigate your claim 


\section{Exploring uncertainty perception as a driver of design activity}

Philip Cash and Melanie Kreye, Department of Management Engineering, Technical University of Denmark, DK-2800 Lyngby, Denmark

This paper investigates uncertainty perception as a general driver of individual design activity. An observation based protocol study is used to explore the interaction between uncertainty perception and three core actions connected in design activity: information action, knowledge sharing action, and representation action. We bring together prior works on uncertainty perception in the design and management literature to derive three contributions. First, we describe how uncertainty perception is associated with activity progression, linking all three core actions. Second, we identify characteristic patterns of interaction between uncertainty perception and activity. Third, we decompose uncertainty perception to further explain its role in driving design activity. This extends prior research on design activity, and supports a number of theoretical and empirical implications.

(c) 2017 Elsevier Ltd. All rights reserved.

Keywords: case study, design activity, design practice, protocol analysis, uncertainty perception

Corresponding author: Philip Cash. pcas@dtu.dk
$\mathrm{D}$ esign activity can be defined as a goal directed system where cognition, behaviour, and motivation are integrated, with respect to the 'bringing-into-being' of a design artefact (Bedny \& Karwowski, 2004; Cash, Hicks, \& Culley, 2015; Dorst \& Cross, 2001). Design activity describes the unity of behaviour and cognition, and its progression determines the performance of the outcome in terms of achieving the design goal (Bilda \& Gero, 2007; DeFranco, Neill, \& Clariana, 2011; Gonçalves, Cardoso, \& Badke-Schaub, 2016; Wasiak et al., 2011). Yet, understanding of the nature of design activity remains limited in two key respects.

First, design activity progression can be decomposed into strings of more fundamental 'core' actions, which are combined in any number of sequential configurations dependant on the subject and situation (Kuutti, 1995). Specifically, the literature describes the central role of three core actions underpinning design activity: information action, knowledge sharing action, and representation action (Cagan et al., 2013; Storga et al., 2010). Each core action has been studied extensively, but in isolation, to identify drivers (Ball \& Christensen, 2009), specific sub-types (Storga et al., 2010), and key outputs (Van Der Lugt, 2005). However, no overall driver, linking the three core

\section{www.elsevier.com/locate/destud}

0142-694X Design Studies $\mathbf{\square}$ (2017)

https://doi.org/10.1016/j.destud.2017.10.004

(c) 2017 Elsevier Ltd. All rights reserved. 
actions, has been described in the literature. Specifically, prior studies have focused on activity progression with respect to a single core action type (within action) as apposed to activity progression linking all three core action types (across action), limiting our ability to describe diverse design activity as observed in practice (Cash et al., 2015).

Second, a common causal mechanism linking cognition and behaviour with respect to the three core actions, fundamental to understanding across action progression, is lacking in current research. Specifically, how a designer's dynamic understanding of a situation is translated into a design goal and subsequently impacts design activity progression is less well understood (Cash et al., 2015; Christensen \& Ball, 2017). Given these limitations a central challenge is identifying an overall driver of design activity, particularly when seeking to explain how and why designers combine various actions in practice to achieve specific outcomes. This poses both theoretical and practical challenges, associated with efforts to develop effective guidance for directing design activity in practice.

Uncertainty perception offers a promising lens from which an overall driver of design activity might be derived (Ball \& Christensen, 2009; Christensen \& Ball, 2017). At the fundamental level, this builds on an understanding of design as a process where uncertainty is gradually resolved (Ball \& Christensen, 2009; Yu, Honda, Sharqawy, \& Yang, 2016). However, before an individual can act on uncertainty, they must perceive and interpret it with respect to their own understanding of the situation (Tversky \& Kahneman, 1974). This link from uncertainty to uncertainty perception and subsequent activity has also been described on a neuro-scientific basis (Alexiou, Zamenopoulos, Johnson, \& Gilbert, 2009). Prior studies have highlighted uncertainty perception as a driver of design activity progression with respect to a number of specific action sub-types, including sketching (Scrivener, Ball, \& Tseng, 2000), and prototyping (Gerber \& Carroll, 2012), and cognitive processes such as mental simulation, co-evolutionary developments in understanding of the problem/solution space (Ball \& Christensen, 2009; Wiltschnig, Christensen, \& Ball, 2013), and creative cognition (Christensen \& Ball, 2017). Thus, this research investigates uncertainty perception as a potential driver of design activity.

In this context, there are two main challenges facing integration of uncertainty perception (Christensen \& Ball, 2017) and design activity (Sim \& Duffy, 2003). First, studies of uncertainty perception in design have typically been experimental and focused on individual action sub-types associated with single core actions (Wiltschnig et al., 2013). For example, prototyping is a specific sub-type of representation action, which is itself only one element of design activity (Cagan et al., 2013). As such, the role of uncertainty perception as a driver of across action design activity progression remains little explored. Second, uncertainty perception in design has been typically characterised as a 
gestalt construct using binary schema (Ball \& Christensen, 2009; Ball, Onarheim, \& Christensen, 2010). This is in contrast to the wider literature where uncertainty perception has been decomposed into a number of dimensions (Hurley, Kosenko, \& Brashers, 2011; O'Connor \& Rice, 2013). As such, the lack of decomposition of uncertainty perception limits descriptive power in the design domain.

In order to resolve these two challenges research must first bridge the different literature dealing with uncertainty perception. Thus, in this work, uncertainty perception is broadly characterised as: a perceived lack of knowledge by an individual, in the form of deficiencies in any stage or activity of the process that can be characterised as not definite, not known, or not reliable (Kreye, Goh, Newnes, \& Goodwin, 2012; Kreye, Newnes, \& Goh, 2012). Building on this, the aim of this study is to explore uncertainty perception as a possible driver for design activity. Specifically, this study investigates dimensions of uncertainty perception as a driver of design activity progression, comprising the three core actions: information, knowledge sharing, and representation. Thus, we explicitly build on and explore the Uncertainty Driven Action (UDA) model proposed by Cash and Kreye (2017). This paper is structured as follows. Section 1 presents a review of research on uncertainty perception and activity from across literature. Section 2 then outlines the empirical study used as the basis for the protocol analysis. Section 3 then reports the results and Section 4 discussion before key implications are derived in Section 5.

\section{Background}

The link between uncertainty perception and activity has received attention in different literature streams; most notable and relevant for this research are design and management. Here uncertainty perception differs from extant uncertainty which describes its nominal existence in a situation, such as organisational and social issues or technology development (Kreye, Goh, et al., 2012; Wynn, Grebici, \& Clarkson, 2011). Uncertainty perception is thus agential because it describes how unsure or unconfident a designer feels based on their perceived lack of knowledge (Kreye, Goh, et al., 2012). In the design literature, perceived lack of knowledge by the individual (i.e. uncertainty perception as defined in this research) has also been referred to as 'epistemic uncertainty' (Ball \& Christensen, 2009), describing a designer's metacognitive awareness of the limitations of their current knowledge or understanding (Ball et al., 2010). In this usage epistemic uncertainty captures a specific instantiation of uncertainty perception as more broadly described in the management literature (Kreye, Goh, et al., 2012). However, epistemic uncertainty in design can also refer to the team or organisation in general, where it is linked to the overall lack of knowledge regarding the problem (Bedford \& Cooke, 2001; Moller \& Beer, 2008; Oberkampf, DeLand, Rutherford, Diegert, \& Alvin, 2002). This second usage captures a distinctly different phenomenon 
and diverges from research connecting uncertainty perception and activity in both literature. As such, for consistency across literature and to avoid confusion within the design literature this research utilises the term uncertainty perception as defined in the introduction. Given this integrative definition, both literature discuss uncertainty perception and its impact on activity, however, contrasts between these bodies of work point to distinct research needs in the design context.

\subsection{Uncertainty perception in design}

Uncertainty is a characteristic feature of design work and has been described as primarily stemming from the unknown nature of the task outcome (Tracey \& Hutchinson, 2016), and the unknown degree to which selected actions will fulfil the designer's goals (Xenakis \& Arnellos, 2013). Here the difference between extant uncertainty and uncertainty perception is particularly important. Uncertainty perception is related to a designer's past experience, attitudes, and personal interpretation (Wiltschnig et al., 2013). Uncertainty perception can thus differ between individual designers (Tracey \& Hutchinson, 2016). Individual uncertainty perception can affect team perception (Fiske \& Taylor, 2008); however this is currently only weakly theoretically described in the design literature. Thus we follow the individual interpretation consistent with prior work on uncertainty perception (Tracey \& Hutchinson, 2016), as well as on design activity (Bedny \& Karwowski, 2004; Sim \& Duffy, 2003). Christensen \& Ball, 2016 illustrate this distinction in their examination of analogising, suggesting that those with greater domain knowledge will perceive and express higher uncertainty. Further, Gerber and Carroll (2012) found that an individual's perception of control and fear of failure mediate their overall perception of and reaction to uncertainty. Thus it is uncertainty perception that is generally recognised as the driver for activity (Ball \& Christensen, 2009). This is typically connected to activity via a process of perception $>$ action $>$ interpretation $>$ new perception, associated with learning (Xenakis \& Arnellos, 2013), where individuals are generally motivated to resolve their uncertainty perception (Bar-Anan, Wilson, \& Gilbert, 2009) via a series of connected actions (Kuutti, 1995).

In design, uncertainty perception has generally been treated as a gestalt construct characterised via binary schema i.e. either present/not present (Ball \& Christensen, 2009; Ball et al., 2010) or low/high (Christensen \& Ball, 2016; Wiltschnig et al., 2013). Change in perception is connected to a situation when individuals are confronted with tasks or stimuli that challenge their existing mental models (Fiske \& Taylor, 2008; Wiltschnig et al., 2013). For example, Gerber and Carroll (2012) describe how uncertainty perception changes when individual's build new knowledge structures. Similarly, Scrivener et al. (2000) show how uncertainty perception changes by the juxtaposition of sketching and an individual's memories. A binary conceptualisation of uncertainty perception may thus miss its fundamental characteristics as a driver of activity. 
Although uncertainty perception as an overall driver of activity can be inferred from the general nature of the construct as highlighted above, research in design has typically focused on specific action sub-types, rather than wider progression across the core actions. For example, Gursoy and Ozkar (2015) show how penmanship and sketching (action sub-types of representation) help to reveal and resolve uncertainty perception while Scrivener et al. (2000) connect sketching and uncertainty perception explicitly in two ways: first, uncertainty perception forms caused strategic shifts within the sketching itself; second, sketching can engender uncertainty perception by elucidating differences between the drawing and the designer's own understanding and memory. Similarly, Gerber and Carroll (2012) highlight how prototyping (action sub-type of representation) also helps to elicit and resolve uncertainty perception by allowing individuals to iteratively build knowledge and promote a sense of control.

More generally, designers create and evaluate possible solution representations in order to gain insight into the problem/solution facing them - reducing uncertainty with respect to the final outcome (Ball et al., 2010; Tracey \& Hutchinson, 2016). These representations can be manifest e.g. in the form of sketches (Scrivener et al., 2000), or latent interpretations of cognition e.g. in the form of mental simulations (Ball et al., 2010). For example, Wiltschnig et al. (2013) describe uncertainty perception as a driver of progression in mental simulation, analogising, and co-evolutionary developments in understanding. Further, uncertainty perception has been linked to information action by authors such as Kim and Lee (2016) and Ulrich and Eppinger (2003) where, designers seek, gather, and interpret information in order to resolve their uncertainty perception. Similarly, knowledge sharing action has been broadly identified as a means of reducing uncertainty perception (Deken, Kleinsmann, Aurisicchio, Lauche, \& Bracewell, 2012; Oppl \& Stary, 2013), stemming from uncertainty about others' understanding of e.g. the solution or task goal (Deken et al., 2012; Kleinsmann \& Valkenburg, 2008). For example, the recent work of Christensen and Ball (2017) highlights the impact of uncertainty perception and creative cognitive processes in group discussions. However, the overall link between uncertainty perception on the progression of design activity (comprising the three core actions) remains unclear.

Based on this review, there are two main limitations connected to current research on uncertainty perception and activity in the design literature. First, uncertainty perception has been characterised predominantly as a gestalt feature with a binary existence scale (Ball \& Christensen, 2009; Ball et al., 2010). In comparison, Lane and Maxfield (2005) decompose uncertainty with respect to truth, semantics, and ontological uncertainty. This suggests that uncertainty perception may have a more nuanced effect on activity progression than currently described. Second, uncertainty perception has been 
shown to drive progression with respect to numerous action sub-types associated with representation (Scrivener et al., 2000), information (Kim \& Lee, 2016) and knowledge sharing (Kleinsmann \& Valkenburg, 2008). However, uncertainty perception has not been described as driving overall across action design activity progression, as observed in practice (Cash et al., 2015) and suggested by theoretical models of design activity as a combinatory construct (Sim $\&$ Duffy, 2003). As such, it is possible to consider the management literature as another relevant source for conceptualising uncertainty perception as a driver of activity.

\subsection{Uncertainty perception in management}

In the management literature, uncertainty perception has been predominantly described as a driver connecting information action and knowledge sharing action (Daft \& Lengel, 1986; Hult, Ketchen, \& Slater, 2004; O'Connor \& Rice, 2013). This has been formalised in models such as Organisational Information Processing Theory (Galbraith, 1974). Here, uncertainty perception is connected to a framework where information is sought then processed through interpretation, reasoning, drawing inferences or learning (Daft \& Lengel, 1983). Information is evaluated through experience and enters the "human belief system as rules for guiding actions' (Song, Van Der Bij, \& Weggeman, 2005) to create knowledge. This individual knowledge is then shared amongst team members to develop joint interpretation and understanding (Berchicci \& Tucci, 2010; Daft \& Lengel, 1986). Thus information action and knowledge sharing action form the basis for reducing uncertainty perception, as in design (Berchicci \& Tucci, 2010). The general characterisation of uncertainty perception as a connected driver of these common actions forms a robust conceptual bridge between the design and management literature, allowing for comparison and cross field learning in this context. In particular, this supports the need to explore uncertainty perception as a driver of across action activity progression.

The management literature highlights the importance of decomposing uncertainty perception to describe its dimensions for driving decision making and activity (Kreye, 2017; Kreye, Newnes, et al., 2012; O’Connor \& Rice, 2013). Specifically, two approaches can be distilled in their relevance for design. First, uncertainty perception can be characterised as a dynamic scalar construct varying from ignorance to certainty (Goh, Newnes, Mileham, Mcmahon, \& Saravi, 2010; Kreye, Goh, \& Newnes et al., 2011). While the design literature remarks that the level of uncertainty perception reduces gradually (Yu et al., 2016), the management literature has highlighted the possibility of uncertainty perception to dynamically vary over the course of a project as individuals' mental models are challenged (Anderson, 1999; Holland, 1995). This dynamic interaction between uncertainty perception and activity indicates the need for a more indepth investigation to determine the specific effect of varying levels of 
uncertainty perception on activity progression. Second, the awareness of uncertainty perception can vary as it can be connected to individuals' systems of thought (Sloman, 2002; Windschitl \& Wells, 1996). For example, implicit uncertainty perception has been associated with the fast and reflexive System 1, while explicit uncertainty perception has been associated with the slow and analytical System 2 (Sloman, 2002; Windschitl \& Wells, 1996). This further emphasises the need for in-depth investigation of the role of uncertainty perception beyond the typical gestalt characterisation found in the design literature.

\subsection{Research focus}

To improve current understanding of design activity and specifically its progression, there is a distinct need to bring together perspectives of design and management. This research examines the role of uncertainty perception as an overall driver of design activity progression across information, knowledge sharing, and representation. This addresses three major questions distilled from the contrasting design and management literature.

First, how does uncertainty perception drive activity progression across the core actions of information, knowledge sharing, and representation? This extends and connects prior studies in the design domain, which have focused on within action design activity progression.

Second, how do dynamic variations in scalar level of uncertainty perception drive across action selection to build up overall design activity progression? This extends prior studies in design, which have used a binary characterisation of uncertainty perception applied with respect to within action design activity progression.

Third, how does the awareness of uncertainty perception, which can be distinguished based on the systems of thought, interact with across action selection to shape overall design activity progression? This again extends prior descriptions in the design literature and connects these to research in the management domain.

The main concepts to be investigated are thus uncertainty perception decomposed with respect to its overall level and the designer's awareness based the systems of thought. This is linked to design activity, connecting the core actions encountered in practice i.e. information, knowledge sharing, and representation.

\section{Methodology}

This research adopts a qualitative approach using an observation study and protocol analysis. This is appropriate for two reasons. First, current literature lacks empirically validated theoretical models of uncertainty perception 
connecting the three core actions, which means there is a need for theory building to advance current understanding in this field (Cross, 2007; Stempfle \& Badke-Schaub, 2002). Specifically, the need to characterise the dynamic interaction between uncertainty perception and activity progression makes an observation based protocol analysis ideal for identifying key features where there is little extant insight (Torgerson \& Torgerson, 2003). Second, protocol analysis has been widely used and demonstrated as effective in the analysis of both design activity (Dorst, 1995) and uncertainty perception (Christensen \& Ball, 2017).

\subsection{Study set-up}

An existing data set was used as the basis for the analysis. This data set has previously been described by Cash, Hicks, and Culley (2013). This approach was used for two main reasons: First, the data set adopted has already been characterised in the design literature and the examined population shown to be representative of industrial practitioners in terms of the core actions undertaken (Cash et al., 2013). This reduces the need for extensive discussion of external validity as this has already been addressed. Second, the data set is publically available which increases the replicability of this research enabling contrasting of findings with other authors also using this data e.g. Martinec, Skec, and Storga (2017).

As the base data set has been extensively described and validated elsewhere (Cash et al., 2013), this section only describes those aspects relevant to the focus of this research as outlined in Section 1.3. The study used an observation set-up, moving participants through the conceptual design stages. This consisted of four sessions (depicted in Table 1) over a total duration of almost $5 \mathrm{~h}$. In the four sessions, the participants moved between individual and team working, linked by the task: 'You are to design a universal camera mount for use on an aerial vehicle. The aerial vehicle is to be used by an amateur photographer, primarily to take still photos.' The participants received no constraints beyond the briefing documents, ensuring that all conversations and activity progression were naturally occurring and were not manipulated by the study facilitator. This was important for the uncertainty perception analysis because

Table 1 The four sessions of the study

\begin{tabular}{|c|c|c|c|}
\hline Session & Duration & Setting & Description \\
\hline 1: Research & $50 \mathrm{~min}$ & Individual & $\begin{array}{l}\text { Given the design task, participants searched for the information } \\
\text { they would need to complete the task. }\end{array}$ \\
\hline 2: Ideation & $50 \mathrm{~min}$ & Team & $\begin{array}{l}\text { Given the information they found in session } 1 \text {, participants } \\
\text { brainstormed possible product designs. }\end{array}$ \\
\hline 3: Development & $90 \mathrm{~min}$ & Individual & $\begin{array}{l}\text { Based on the ideas generated in session } 2 \text {, participants developed } \\
\text { one idea further to prototype level of detail. }\end{array}$ \\
\hline 4: Review & $50 \mathrm{~min}$ & Team & $\begin{array}{l}\text { Based on the three prototype-level designs from session } 3 \text {, } \\
\text { participants discussed their feasibility and chose one final concept. }\end{array}$ \\
\hline
\end{tabular}


of the constraints of content analysis in coding natural language (Krippendorff, 1981). Specifically, this research focuses on two of the four sessions (ideation and review) because these were team working session that contained natural verbalisations tractable through content analysis. The remaining two sessions were individual working sessions that did not include any verbalisations relevant for this research. Data was recorded via multiple cameras, enabling a full recording of discussions, note taking, sketching, whiteboard use, and physical gestures/expressions.

Twelve participants were selected from a final year master's product design course at a UK-based university. This provided a relatively homogenous population suitable for the detailed behavioural analysis required for this study. The participants were highly motivated and familiar with each of the core actions i.e. having training and experience in information action, knowledge sharing action, and representation action. The twelve participants were randomly allocated to four teams and provided with all the materials required to complete the design task.

\subsection{Data analysis}

The unit of analysis of this research was the individual designer. The data analysis focussed on uncertainty perception and core actions. Uncertainty perception was coded using content analysis (Krippendorff, 1981) which focuses on reducing a large amount of text into fewer categories (Weber, 1990). This enabled us to obtain quantitative measures through rigorous and explicit rules of coding. Content analysis ensures reliability, validity, and repeatability (Krippendorff, 2004; Neuendorf, 2002). This approach further aligns with a previously validated approach in the design community (Ball \& Christensen, 2009; Ball et al., 2010; Christensen \& Ball, 2017; Wiltschnig et al., 2013). It was thus suitable for this research.

Content analysis was applied in two steps. In the first step all words or phrases linked to uncertainty perception were coded at the individual level using a content analysis approach. Content analysis is typically applied by measuring a specific concept through 'counting the occurrences of meaning units such as specific words' (Weber, 1990). A set of words and short phrases was used as the basis for this first step, which was drawn from the psychology, management, and design literature where the terms were developed from previously reported links between statements and uncertainty perception (see Table 1, in this step a combination of terms from implicit and explicit uncertainty perception were used). This delivered an overall count of uncertainty expressions to describe the aggregate level of uncertainty perception (Hurley et al., 2011). Here the analysis focused on changes in level rather than the overall level itself. This builds on the underlying logic that it is dynamic changes in uncertainty perception that drive activity progression (Wiltschnig et al., 2013). 
In the second step overall uncertainty perception was decomposed in order to provide a more nuanced description of the changes in uncertainty perception with respect to the designer's awareness. Specifically, we differentiated explicit uncertainty statements, implicit statements, and explicit certainty statements (negation of uncertainty). The coding approach for these categories included initial validation on a randomly selected subset of the data to ensure data reliability. This is a suitable approach as described in the content-analysis literature (Krippendorff, 2004; Neuendorf, 2002). The coding was done manually to ensure that the use of terminology was aligned with the coding structure. Examples of the terminology use were: 'this [product design] makes sense' (negation), 'perhaps we could explore [another design option]' (Implicit uncertainty), and 'I don't know how this would work' (explicit uncertainty). The full list of terms and sources used for coding uncertainty perception is outlined in Table 2, distributed with respect to explicit, implicit, and negation. Each of these decomposed dimensions of awareness of uncertainty perception were again assessed with respect term frequency.

Core actions were coded at the individual level based on previously validated schema as follows. As each of the coding schemas used for the various actions included below have been described in various other design research they were considered to be already robust and reliable. For example, information action corresponds to an aggregation of specific codes from Wasiak, Hicks, Newnes, Dong, and Burrow (2010), Robinson (2010), and Deken et al. (2012), which have all individually been validated in the design context. In particular, this approach was adopted in order to focus on uncertainty perception and reduce the need for extensive discussion of the design activity schema.

Information action: dealing with data parts and their manipulation e.g. collection, recording, reviewing, filing, archiving, seeking, and requesting (Deken et al., 2012; Robinson, 2010; Wasiak et al., 2010). This captured actions including searching for data online, or providing/asking for specific data. Information action explicitly deals with the manipulation of data parts and orienting statements of fact detached from an individual's beliefs (Belkin, Oddy, \& Brooks, 1982; Song et al., 2005).

Knowledge sharing action: dealing with exchanging knowledge with the design team, to develop a joint interpretation and understanding in a group (Deken et al., 2012; Kleinsmann \& Valkenburg, 2008). This captured actions including informing other people in the design team about an individual's opinion, resolving issues or clarify problems, evaluating or expressing a preference, and giving direction or possible modes of action (Bales, 2008). Knowledge sharing action is explicitly linked to an individual's own understanding and beliefs expressed via opinion and suggestion, distinguishing it from information action (Chiu, Hsu, \& Wang, 2006; Song et al., 2005). 
Table 2 Terms associated with the different dimensions of decomposed uncertainty perception

Dimension of uncertainty Terms included with exemplar references

Explicit

Uncertain, uncertainty; risk, risky (Bedford \& Cooke, 2001); variation, vary, variable (Walker et al., 2003); chance (Langer \& Roth, 1975); confident, confidence, not confident (Lawrence \& Makridakis, 1989); imprecise, imprecision (Arad \& Gayer, 2012); vague, vagueness, vaguely (Ellsberg, 2001); ambiguous, ambiguity (Ellsberg, 2001); $\mathrm{x} \%$, probable, probability, probably (Bedford \& Cooke, 2001); likely, unlikely (Kreye, Goh, et al., 2012); uncertainty modelling techniques such as Sensitivity analysis or Monte Carlo (Beynon, Curry, \& Morgan, 2000); unknown, not known, don't know (Soanes, 1928); ignorance, ignore, ignorant (Walker et al., 2003); Interval statement (e.g. maximum, minimum, worst case, best case, biggest, smallest, heaviest, lightest) (Kreye, Goh, et al., 2012); on average, mean, around (Langer \& Roth, 1975)

Implicit Re- (redo, renegotiate, reschedule etc) (Shafer, 1994); mis- (miscommunicate, misunderstand etc) (Berger, Gudykunst, \& Deryin, 1991); change, changed (Song \& Montoya-Weiss, 2001); maybe, perhaps (Hurley et al., 2011); expected, expect, expectation, expectedly, unexpected (ISO, 2009); possible, impossible, not possible, possibly (Hurley et al., 2011); potential, potentially (Soanes, 1928); if ... then, in case, depending on, depend on, alternative, alternatively, otherwise (Anderson, Holmes, \& Good, 1973); different, differently (Kreye, Goh, et al., 2012); suggest, suggestion, suggested (McKenna, 2002); almost, most (Kreye, Goh, et al., 2012); undecided, not decided (Sicotte \& Bourgault, 2008); predict, forecast, estimate (Goodwin \& Wright, 1993); guess, think, wonder, thought, reckon, imagine (Morone \& Morone, 2008); may, could, can, might (Hurley et al., 2011); suppose, supposed to, supposedly (Harvey, 2001); assume, presume, presumably, presumed (Kreye, Goh, et al., 2012); lack of ..., not enough, missing (knowledge, information, data etc.) (Zimmermann, 2000); available (data, information, evidence etc.) (Hand \& Walley, 1993); confusing, confused, confusing (Hurley et al., 2011); experience, inexperience, inexperienced (Beresnev, Shestirko, \& Nazarenko, 1983); vagueness in statement (some sort of ..., seem to ...) (Berger et al., 1991); not sure, unsure (Kreye, Newnes, et al., 2012); not clear, unclear, clarify, clarification, clarity (Ellsberg, 2001); not defined, undefined (ISO, 2009); common, not common, commonly, usually, usual, typically, typical (Kreye, Goh, et al., 2012); disagree, disagreement, not agree (Hand \& Walley, 1993); not understand, not understood (ISO, 2009)

Negation Certain, sure, assured, assurance (Kreye, Goh, et al., 2012), precise, precisely, exact, exactly, well known, well understood, well defined (Soanes, 1928); definite, definitely, absolutely, absolute (Kreye, Goh, et al., 2012); agree, make sense (Hand \& Walley, 1993); clear, clearly (Ellsberg, 2001); never, always (Kreye, Goh, et al., 2012)

Representation action: dealing with the manipulation of external information representations (Cagan et al., 2013; Cash \& Maier, 2016). This captured actions including sketching possible product designs on the whiteboard, prototyping, and gesturing product functionalities (Sanders \& Stappers, 2014; Schön \& Wiggins, 1992). Representation actions is explicitly linked to the interplay between an individual's internal and external representations (Scaife \& Rogers, 1996; Wiltschnig et al., 2013), distinguishing it from the other actions.

Krippendorff's alpha (Krippendorff, 1981) was calculated for the core action codes using two raters on a data sample with continuous coding intervals of $1 \mathrm{~s}$. This resulted in alpha values of $0.93,0.95$, and 0.98 for information action, knowledge sharing action, and representation action respectively. The

Uncertainty perception and design activity 
uncertainty perception terms were evaluated via percentage agreement. Here, $100 \%$ agreement was reached due to the manifest nature of the schema.

\section{Results}

The results are presented with regard to the changes in aggregate level of uncertainty perception before detailing the decomposition of awareness of uncertainty perception.

\subsection{Aggregate level of uncertainty perception and activity progression}

The results for each individual study participant were mapped with regard to uncertainty perception and activity progression with respect to the sequential combination of the three core actions. This section describes the results for an example participant; however, the results of this analysis included all participants. Figure 1 shows the results for an example participant for both the ideation and review sessions (Table 1) with the time axis in seconds. Activity progression (across the core actions) is shown using a percentage of a $60 \mathrm{~s}$ moving average. This is juxtaposed with change in aggregate level of uncertainty perception. Change in perception was used in order to better illustrate the dynamic nature of the interaction between uncertainty perception and activity. From this analysis, two main findings were derived, showing uncertainty perception as a driver of within and across action design activity progression.

Focusing on within action design activity progression, there are a number of periods within the dataset where participants work almost exclusively with one core action (information, knowledge sharing, or representation). This observation was associated with changes in aggregate level of uncertainty perception $(\Delta$ LUP). Uncertainty perception generally increased at the start of the action, decreased as the action progressed, and increase again when changing strategy within the action, before being gradually resolved to baseline levels at the end of the progression. It is important to note that although increasing uncertainty perception was connected to such progressions, it was also evident that progression could be connected to decreasing uncertainty perception. Thus, within action design activity progression was affected by 'change' in level of uncertainty perception ( $\Delta$ LUP). A prototypical within action activity progression can thus be described in generic terms, and is illustrated in Example 1 as follows:

Prototypical within action design activity progression: $\boldsymbol{\Delta L U P}>$ starting action $>$ action caused $\triangle L U P>$ within action change of strategy $>$ action caused $\triangle L U P>\ldots>\Delta L U P$ to baseline $>$ end

Example 1: Within action design activity progression; Team 1, Participant 2, Ideation, $17.30-20.00 \mathrm{~min}$ 

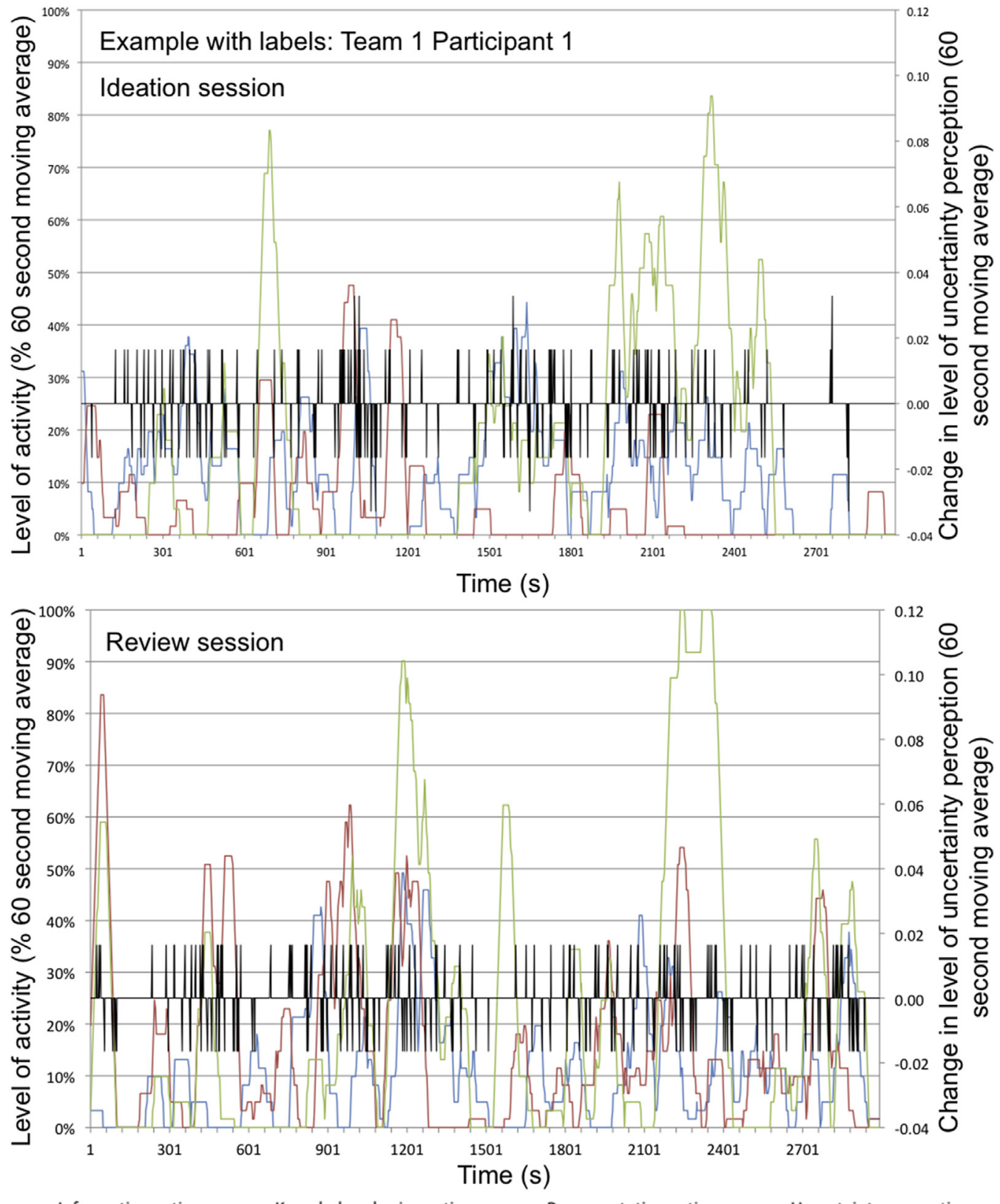

Figure 1 Change in aggregate level of uncertainty perception and activity progression for example participant in ideation and review

In observing the activity progression in this example, three distinct phases emerged (see Figure 2). Phase 1 started with information action where Participant 2 (P2T1) provided information to the team based on their research findings. These results were questioned by the other participants with respect to the context in which they were considered e.g. the worst case scenario. Here, 
$\mathrm{P} 2 \mathrm{~T} 1$ added into the discussion between $\mathrm{P} 1 \mathrm{~T} 1$ and $\mathrm{P} 3 \mathrm{~T} 1$, providing concrete facts to complement their more speculative discussion. Here we observed a rapid change in uncertainty perception as illustrated in Figure 2, increasing and decreasing as questions were raised and answered. In the end discussion about the camera weight was resolved, which was connected to a shift in topic to camera size. This was associated with P2T1 taking the lead in the discussion and giving a series of details from their research findings, followed by answering specific questions from the team in the second half of this phase. Phase 2 was characterised by a distinctly different strategy in the information action coupled with the sequential increase and then resolution of uncertainty perception. Finally, the activity progression came to a close (Phase 3 ) as the research information was clarified and level of uncertainty perception was gradually reduced to a natural end point in the discussion of camera type. Here, P2T1 added information explicitly into a more judgement orientated discussion between P1T1 and P3T1, helping them to formalise the previous discussion points.

\begin{tabular}{llll}
\hline Time & Action & Person & \multicolumn{1}{c}{ Explanation } \\
\hline 17.30 & Information (Phase 1) & P2T1 & $\begin{array}{l}\text { Informing the team about their findings on camera weight } \\
\text { from their research, contributing to a wider discussion }\end{array}$ \\
18.20 & Information (Phase 2) & P2T1 & $\begin{array}{l}\text { Listing specific details about the size of possible cameras, } \\
\text { leading the team in an informing question and answer period } \\
\text { Adding information into a wider discussion between P1T1 and P3T1 }\end{array}$ \\
19.20 & Information (Phase 3) & P2T1 & Adwen
\end{tabular}

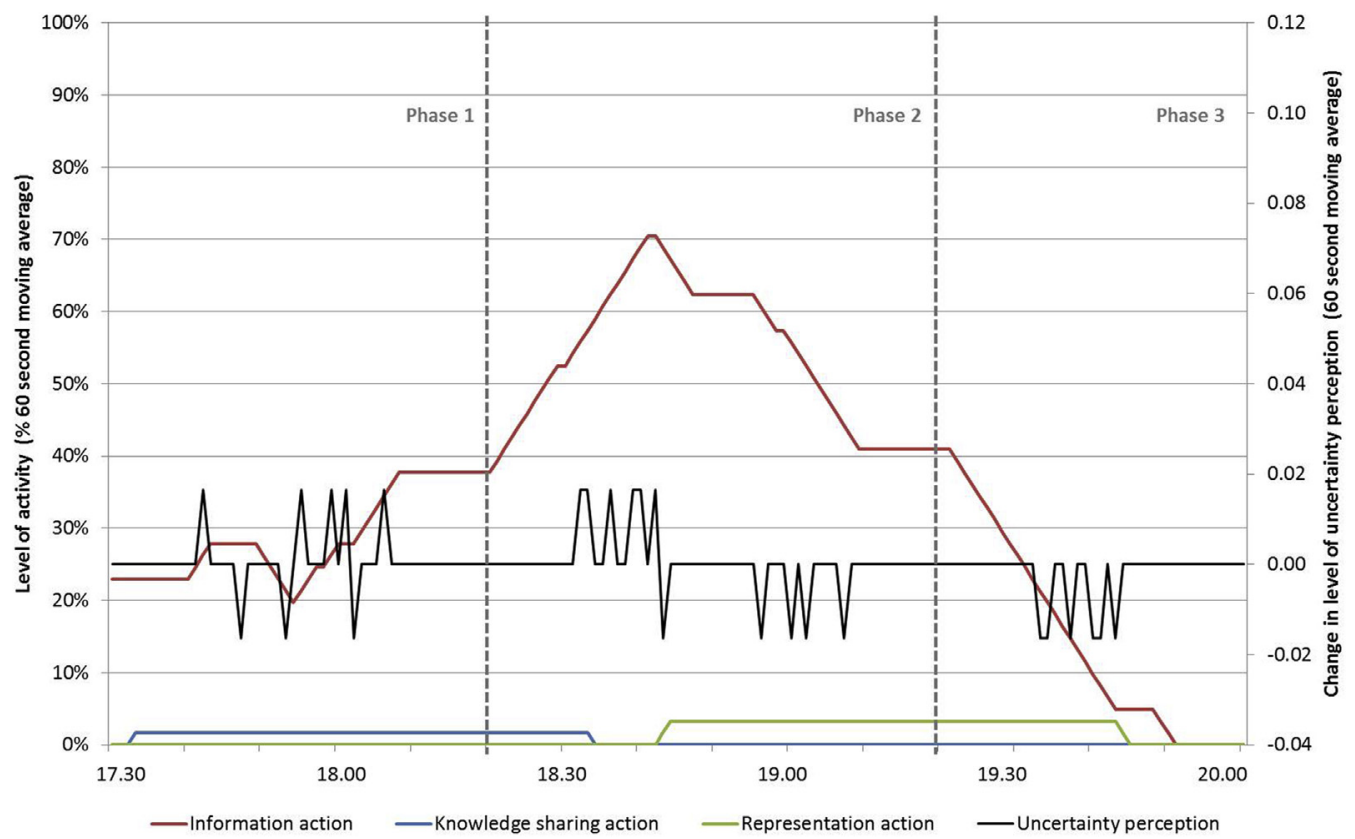

Figure 2 Detail of within action design activity progression for Example 1 
Focusing on across action design activity progression, changes in aggregate level of uncertainty perception were again found to be critical to shifts across information, knowledge exchange, and representation. Thus the second main finding at this level of analysis is that changes in aggregate level of uncertainty perception drive across action design activity progression. Shifts across actions were found to be associated with both increases and decreases in aggregate level of uncertainty perception, again highlighting dynamic change as the cause for progression. Although, increasing uncertainty perception appears to be the more common driver, the period of activity illustrated in Example 2 was connected to reduction of uncertainty perception. Again, a prototypical across action activity progression can be described in generic terms, and is illustrated in Example 2 as follows:

Prototypical across action design activity progression: $\boldsymbol{\Delta L U P}>$ starting action $>$ action caused $\triangle L U P>$ change of action $>$ action caused $\triangle L U P>\ldots>\Delta L U P$ to baseline $>$ end

Example 2: Across action design activity progression; Team 3, Participant 2, Review, 17.00-19.30 min

In this example there was a progression linking information, knowledge sharing, and representation. This was preceded by reduction in the level of uncertainty perception that was connected to the team's agreement that now is the time to start drawing up their concept. Participant 2 (P2T3) started the progression by alternating between sketching the final concept and informing the other participants about the design - this was initially associated with reducing uncertainty perception in Figure 4. However, as the sketch progressed a number of problems with the concept were revealed which was associated with an increase in uncertainty perception. This then caused a transition to knowledge sharing action, again supported by alternating representational sketching. Here, P2T3 perceived uncertainty regarding the housing and overall integration between the mounting and camera. This prompted P3T3 and P1T3 to exchange ideas and opinion about the concept. This was characterised by an

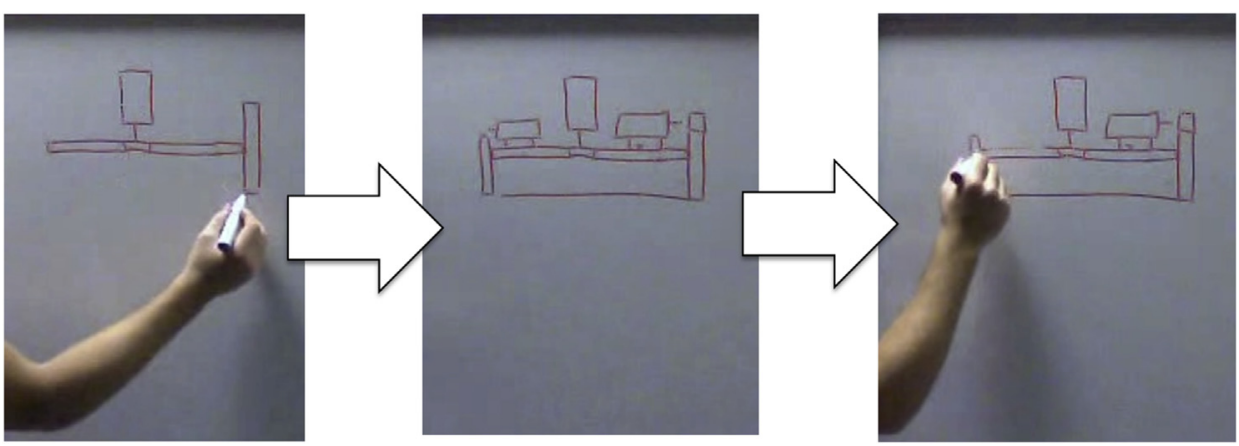

Figure 3 Progression of sketched representation of the final concept for Team 3

Uncertainty perception and design activity 
intense period of alternative representation and knowledge sharing action as P2T3 iteratively develops the sketch and attempts to address the opinions from the team. In particular, sketch elements were added and then removed as the knowledge sharing progressed (see the transition between Figure 3 part 2 and part 3). This alternating progression and iteration of the concept sketch based on the knowledge sharing action was again associated with alternating increasing and decreasing uncertainty perception. Finally, the knowledge sharing action reduced and the progression was concluded by representation action where the team agreed a final configuration. This final period of agreement and resolution was associated with reduction in the level of uncertainty perception as illustrated in Figure 4.

\begin{tabular}{|c|c|c|c|}
\hline Time & Action & Person & Explanation \\
\hline 17.00 & $\begin{array}{l}\text { Information/ } \\
\text { representation (Phase 1) }\end{array}$ & P2T3 & $\begin{array}{l}\text { Sketching a concept and verbalising its working } \\
\text { principles (Figure } 4 \text { part 1) }\end{array}$ \\
\hline 17.40 & Knowledge sharing & P3T3 & $\begin{array}{l}\text { Suggests a new idea for the concept housing and P1T3 } \\
\text { gives opinion about 'where the camera fits in the housing' }\end{array}$ \\
\hline 17.50 & $\begin{array}{l}\text { Knowledge sharing/ } \\
\text { representation (Phase 2) }\end{array}$ & P2T3 & $\begin{array}{l}\text { Develops the sketch to address the suggestion and } \\
\text { opinion, as well as elaborating the motor placement, } \\
\text { this alternates with explanations directed specifically } \\
\text { to the other participants (Figure } 4 \text { part 2) }\end{array}$ \\
\hline 19.00 & $\begin{array}{l}\text { Knowledge sharing }> \\
\text { representation (Phase } 3 \text { ) }\end{array}$ & P2T3 & $\begin{array}{l}\text { Concludes a solution for balancing the housing, } \\
\text { given the developments made in the sketch (Figure } 4 \\
\text { part 3) and finalises the representation of the concept }\end{array}$ \\
\hline
\end{tabular}

\subsection{Decomposed uncertainty perception and activity progression}

Decomposing uncertainty perception into explicit, implicit and negation and their impact on activity progression showed complex and varied interactions with three general features. First, negation was often associated with a change of topic (i.e. participants were certain about something) and a subsequent change in action associated with the new topic. In other words, negation occurred typically either at the very start or very end of a progression with few instances observed mid sequence. Second, implicit uncertainty was the most consistent component associated with design activity progression and was found to be evident in all periods of activity, following the patterns of progression described in Section 3.1. Third, implicit uncertainty was found to connect to explicit uncertainty in a number of distinct ways. For example, explicit uncertainty often concluded an activity progression, where numerous prior implicit uncertainty statements were formalised in agreed rationalisations. Thus, explicit uncertainty was observed in a similar role to negation, concluding an activity progression and causing a new sequence of actions. Explicit uncertainty was also observed in this formalisation context at points of across action design activity progression, unlike negation. Less expected, 


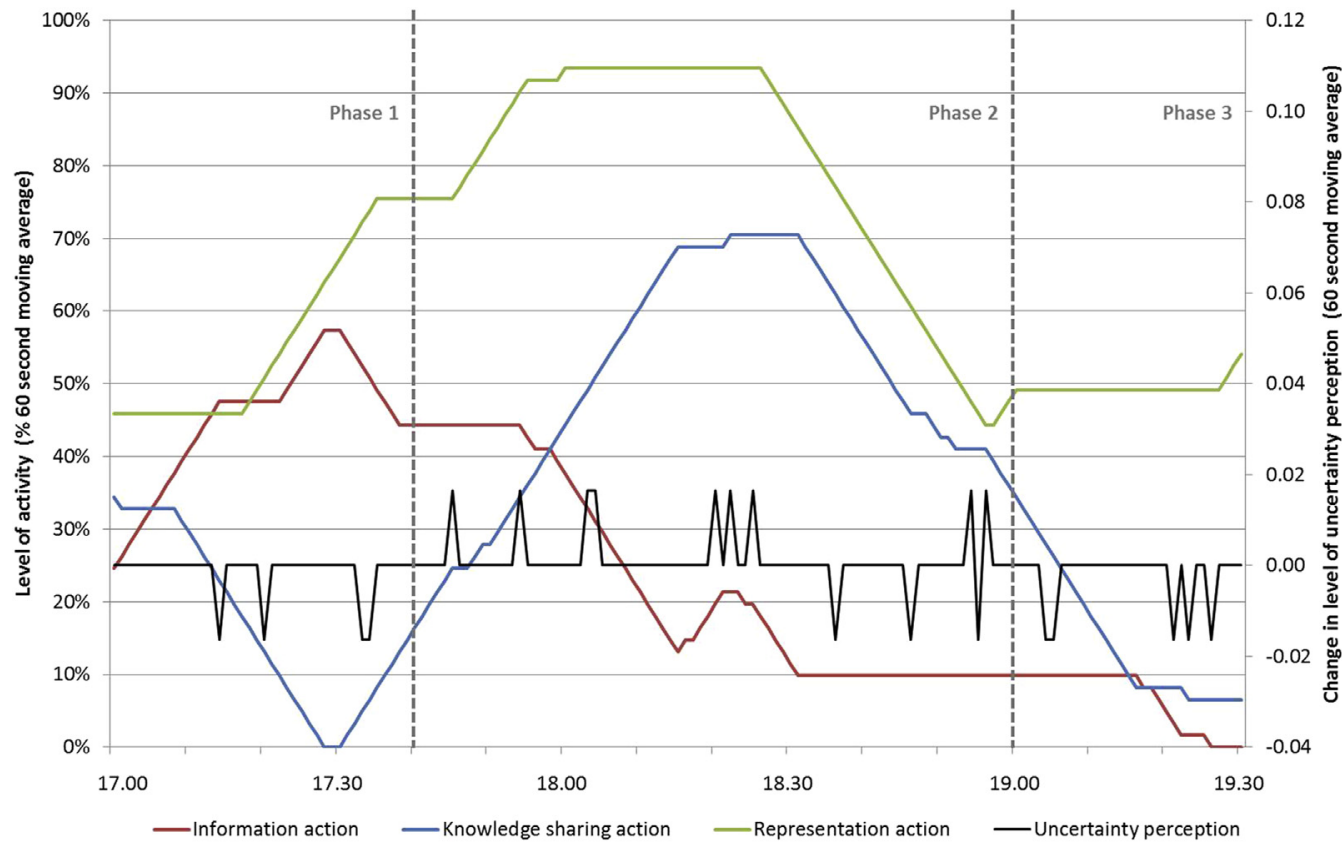

Figure 4 Detail of across action design activity progression for Example 2

however, was that in a number of instances explicit uncertainty preceded implicit uncertainty and acted more as a seed for elaboration i.e. an explicit expression was followed by implicit judgements and explanation. A number of these features are illustrated in Example 3.

Example 3: Decomposing uncertainty perception; Team 1, Participant 1, Ideation, $16.00-20.00 \mathrm{~min}$

Example 3 elaborates the segment from Example 1 with respect to Participant 1 (P1T1). In this segment P1T1 progressed through a number of connected information and knowledge sharing actions following different dimensions of uncertainty perception. The period started with a decrease in implicit uncertainty and increase in negation as a topic of discussion came to a close and final details were agreed via knowledge sharing (specifically the drone power specifications). This decreased uncertainty perception was connected to a shift in topic of discussion, action (to information), and uncertainty perception (increasing explicit followed by implicit uncertainty). Subsequent to this P1T1 gave a wide range of research information to the team where a number of explicit and implicit uncertainties were expressed. In particular these typically began with the presentation of explicit uncertainty linked to a research finding, followed by elaboration of the implications of these findings associated with implicit uncertainty. Characteristic of this period were explicit uncertainty, followed by implicit uncertainty as the initial fact was elaborated and 
less concrete dimensions were discussed. This then caused a shift to knowledge sharing action as the discussion refocused on judgements about the research findings. This was associated with fluctuation in both explicit and implicit uncertainty, characterised by a mix of explicit statements e.g. 'Camera type $X$ ranges in weight up to $4 \mathrm{~kg}$ ', elaborated by implicit statements e.g. 'Camera type $X$... so it would likely not be owned by amateur photographers, so around $1-1.5 \mathrm{~kg}$ for an amateur I would imagine'. As this action came to a close implicit uncertainty perception decreased and explicit uncertainty perception increased. Finally, in the last phase of this example P1T1 returned to their research notes, which again illustrates the pattern of explicit followed by implicit uncertainty perception. The interaction between decomposed uncertainty perception and activity progression during the example period is illustrated in Figure 5.

\begin{tabular}{|c|c|c|c|}
\hline Time & Action & Person & Explanation \\
\hline 16.00 & $\begin{array}{l}\text { Knowledge sharing }>\text { information } \\
\text { (Phase 1) }\end{array}$ & P1T1 & $\begin{array}{l}\text { The team is discussing the specification of the drone, } \mathrm{P} 1 \text { is } \\
\text { agreeing with the identification of batteries as a power } \\
\text { source, reading from their research on batteries and } \\
\text { confirming questions from P2T1 }\end{array}$ \\
\hline \multicolumn{4}{|c|}{ Decreasing implicit perception and increasing negation perception } \\
\hline 16.30 & Information (Phase 2) & $\mathrm{P} 1 \mathrm{~T} 1$ & $\begin{array}{l}\text { Informing the team about their findings on camera } \\
\text { specifications, P1T1 initially states facts before giving } \\
\text { opinion based judgements about the scope of the } \\
\text { requirements }\end{array}$ \\
\hline \multicolumn{4}{|c|}{ Increasing implicit perception following increasing explicit perception } \\
\hline 17.10 & $\begin{array}{l}\text { Information }>\text { knowledge sharing } \\
\text { (Phase 3) }\end{array}$ & P1T1 & $\begin{array}{l}\text { Discussing the context of the results i.e. 'considering the } \\
\text { worst case scenario.' }\end{array}$ \\
\hline \multicolumn{4}{|c|}{ Fluctuating explicit and implicit perception } \\
\hline 18.00 & knowledge sharing (Phase 4) & P1T1 & $\begin{array}{l}\text { Offering judgements and elaborating on the information } \\
\text { presented by P2T1 }\end{array}$ \\
\hline \multicolumn{4}{|c|}{ Decreasing explicit perception following decreasing implicit perception } \\
\hline 19.00 & Information (Phase 5) & $\mathrm{P} 1 \mathrm{~T} 1$ & $\begin{array}{l}\text { Returns to research findings and enumerates a number of } \\
\text { results for the team }\end{array}$ \\
\hline \multicolumn{4}{|c|}{ Implicit perception following explicit perception } \\
\hline
\end{tabular}

\subsection{Robustness of decomposed uncertainty perception}

Finally, a number of checks were carried out in order to assess the robustness of the decomposed uncertainty perception schema (Table 2). These assessed its validity at three levels: action, participant, and session. First, in terms of the different actions observed, there were a number of significantly strong positive correlations with the core actions (information, knowledge sharing, and representation). This is despite the small sample size and the fact that representation action is primarily non-verbal and thus less subject to issues of endogeneity. This correlation analysis is outlined in Table 3, where correlation and significance were tested with respect to Pearson's correlation coefficient (r). Although this study is qualitative and thus these 


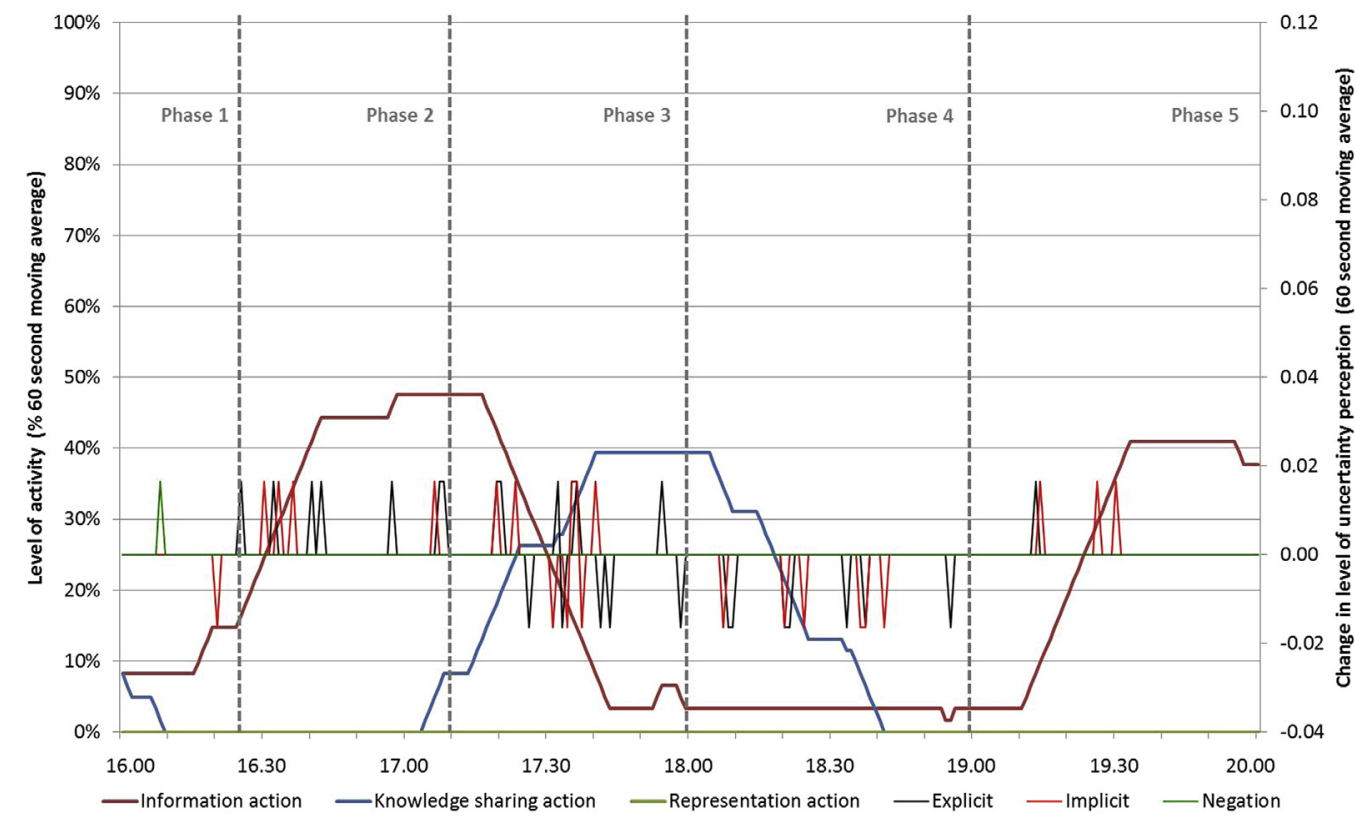

Figure 5 Detail of decomposed uncertainty perception and activity progression for Example 3

results should not be taken as generalizable they do suggest that the data set is valid for the purposes of this work, and that the findings associated with decomposed uncertainty perception might be suitable for generalisation in future quantitative studies.

Second, differentiation in terms of the decomposed dimensions of uncertainty perception was significant across participants $(\mathrm{p}<0.01$, two tailed Students t-test). Of all the uncertainty expressions used, explicit accounted for an average of $9 \%(\mathrm{SD}=4 \%)$, implicit $89 \%(\mathrm{SD}=4 \%)$, and negation $2 \%(\mathrm{SD}=1 \%)$. Third, this distribution showed no significant differences when compared across sessions (ideation and review). The robustness of these findings was further checked by carrying out the same analysis after normalising for the number of coded terms in each category, as well as normalising for the amount of time each participant spent talking. In both cases the results remained significant.

Table 3 Correlation between decomposed uncertainty perception and the elements of design activity

\begin{tabular}{llll}
\hline Uncertainty code & & Action code & \\
\cline { 2 - 4 } & Information & Knowledge sharing & Representation \\
\hline Explicit & $\mathrm{r}=0.50 \mathrm{p}=0.097$ & $\mathrm{r}=0.44 \mathrm{p}=0.156$ & $\mathrm{r}=0.22 \mathrm{p}=0.487$ \\
Implicit & $\mathbf{r}=\mathbf{0 . 6 4} \mathbf{p}=\mathbf{0 . 0 2 4}$ & $\mathbf{r}=\mathbf{0 . 8 8} \mathbf{p}=\mathbf{0 . 0 0 0}$ & $\mathrm{r}=0.51 \mathrm{p}=0.092$ \\
Negation & $\mathrm{r}=0.33 \mathrm{p}=0.288$ & $\mathbf{r}=\mathbf{0 . 7 1} \mathbf{p}=\mathbf{0 . 0 0 9}$ & $\mathbf{r}=\mathbf{0 . 6 3} \mathbf{p}=\mathbf{0 . 0 2 7}$ \\
\hline
\end{tabular}

Bold text highlights significant $(\mathrm{p}<0.05)$ correlations. 
Based on these robustness checks, it is possible to conclude that despite participant level differences in amount of overall uncertainty perception, amount of total time spent speaking, and amount of time spent on different actions, the decomposition of uncertainty perception used in this study forms a robust characterisation across individuals and situations - supporting its fundamental link with the systems of thought. Thus, uncertainty perception may be a general driver of activity progression in this study.

\section{Discussion: uncertainty perception as a general driver of design activity progression}

The presented findings show the importance of uncertainty perception in driving design activity progression, specifically illustrating uncertainty perception's role in shaping the sequential combination of all three core actions. The findings showed that uncertainty perception, both as an aggregated and as a decomposed construct were connected to the progression of design activity, shaping both within and across action design activity progression. Further, the design literature has described the possible role of uncertainty perception in linking specific aspects of cognition and behaviour (Ball, St.B.T. Evans, Dennis, \& Ormerod, 1997; Deken et al., 2012; Wiltschnig et al., 2013). The study findings elaborate these descriptions by evidencing uncertainty perception as a potential causal mechanism driving the progression of design activity. Specifically, two main findings can be derived based on the presented study associated with design activity progression with respect to a single core action type (within action) and design activity progression linking all three core action types (across action).

The findings with regard to within action design activity progression confirm prior research (Christensen \& Ball, 2017; Scrivener et al., 2000). The type of activity progression illustrated in Example 1 broadly follows those results previously described by, for example Scrivener et al. (2000). Further, they conform with many of the features of within action design activity progression described by Christensen and Ball (2017). For instance, Example 1 shows how changes in level of uncertainty perception were associated with information elicitation and selection connected to camera weight, and camera size/use scenario. Further, the participant expressed heightened uncertainty perception associated with distinct phases within the progression. In the transition between these phases, the participant changed their disposition within the team as well as the sub-type of action being undertaken (e.g. moving from gesture to sketching; action sub-types of representation). As such, these findings strongly support the general assertion that changes in aggregate level of uncertainty perception are a driver of within action design activity progression (Christensen \& Ball, 2016; Wiltschnig et al., 2013), and are associated with changing strategies dependant on participant experience and own understanding (Scrivener et al., 2000). This supports the overall validity of the results 
reported here and suggests that they are comparable with prior studies in the design domain (Christensen \& Ball, 2017).

The findings with regard to across action design activity progression highlight a number of key features of the dynamic interaction between aggregate level of uncertainty perception and activity progression not previously described in the design literature. The initial action arose from a decrease in uncertainty perception precipitating a change of topic and thus a new progression. This elaborates prior conceptualisations of the relationship between uncertainty perception and design activity. The literature has focused on increasing uncertainty perception as a driver for within action design activity progression (Scrivener et al., 2000; Wiltschnig et al., 2013). The results here point towards a more neutral interpretation where change in uncertainty perception, both positive and negative, drive both within and across action design activity progression.

Changes in uncertainty perception drove progression linking the three core actions (information, knowledge sharing, representation) following a similar structure to that described with respect to within action design activity progression (Christensen \& Ball, 2017). The nature of these transitions suggest that they are underpinned by the latent cognitive processes linked to uncertainty perception by Christensen and Ball $(2016,2017)$ e.g. mental simulation, analogising, and co-evolutionary development in understanding. These results also align with and extend the findings of Scrivener et al. (2000). For example, representational sketching was found to confront participants with uncertainties not previously considered and thus drove across action activity progression, much as it drove within action activity progression in Scrivener et al.'s study.

Here, the decomposition of uncertainty perception provided further insights beyond existing descriptions that significantly extend prior characterisations of the relationship between uncertainty perception and design activity. The findings suggest an opposing transition from implicit to explicit uncertainty perception. This finding is relatively surprising with respect to prior works specifically focusing on uncertainty perception where the opposite transition is highlighted (Sloman, 2002). However, it generally conforms to known patterns of activity in design. For example, elaboration on concrete inspirational elements via opinion and judgement has been previously described with respect to ideation by authors such as Gonçalves et al. (2016). The finding of an associated transition from explicit to implicit uncertainty perception supports prior research findings and the validity of the other insights reported here. In particular, this feature of explicit formalisation following implicit discussion fits well with prior conceptualisations of these dimensions in the literature (Kreye, 2013). 
Despite the identification of a number of characteristic patterns associated with decomposed uncertainty perception and design activity progression, this interaction remains a complex issue. In particular, questions remain as to how the different proportional combinations of negation, explicit, and implicit uncertainty influence the nature of activity progression, particularly with respect to how it shapes the sequential combination of core actions. For example, it is not clear if one combination of uncertainty perception would predict the selection of one particular core action over another. However, it is widely assumed that such predictive relationships do exist, and play a role in directing activity progression in the management literature (De Brentani \& Reid, 2012; Downey \& Slocum, 1975; O'Connor \& Rice, 2013). In this understanding, changes in the compositional nature of uncertainty perception also drive within and across action design activity progression. Thus, a generic conceptualisation of the interaction between uncertainty perception and design activity progression is illustrated in Figure 6. Here large changes in uncertainty perception (level or composition) drive across action activity progression while more moderate changes drive within action activity progression. Figure 6 is to be understood as a conceptual representation of our core findings not as a depiction of results with measurements. In particular, such a normalised-results-based representation is not currently possible due to the individual differences in timescale, magnitude, and response associated with the

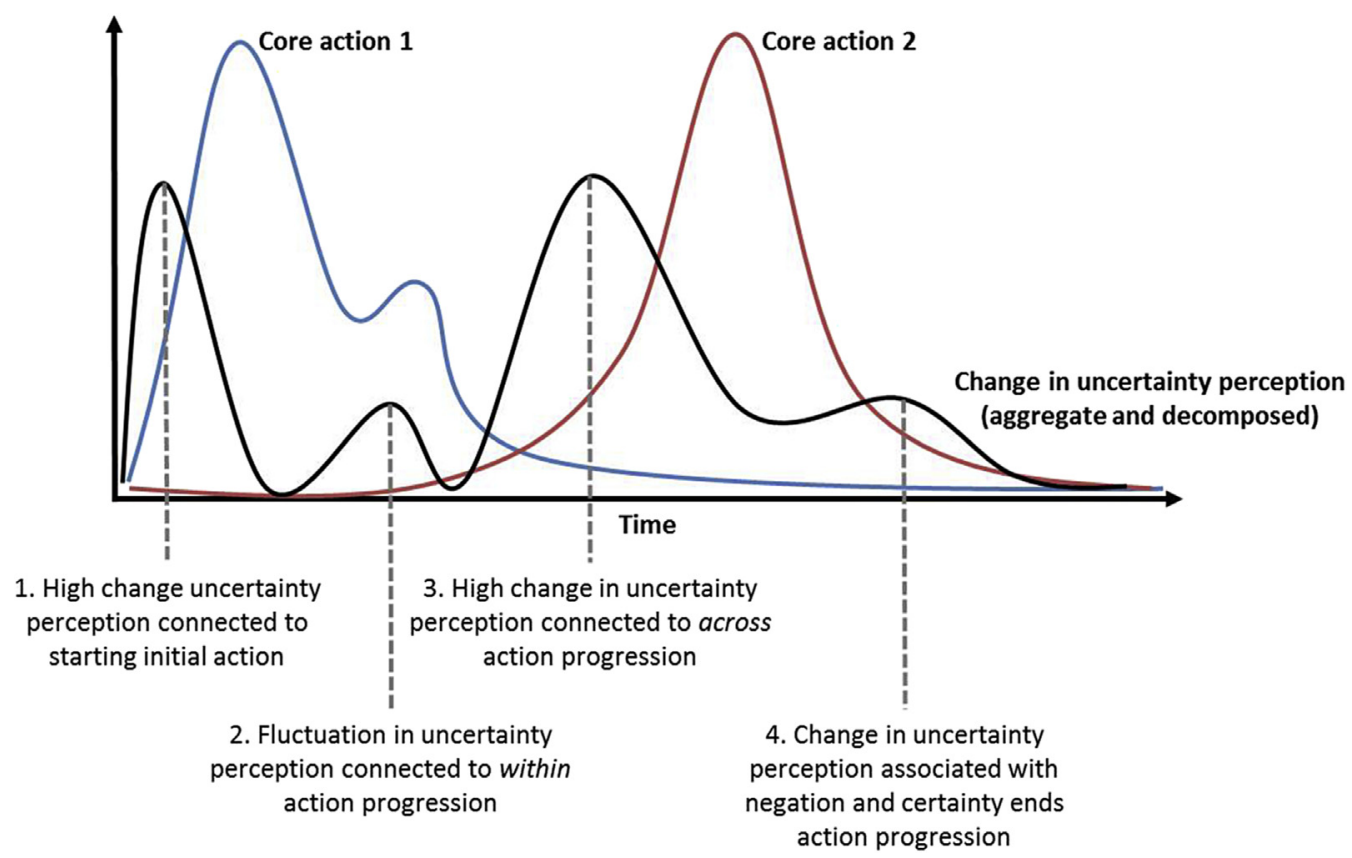

Figure 6 Generic conceptualisation of the interaction between change in uncertainty perception (level or composition) and design activity progression comprising the three core actions 
perception/activity interaction. Thus, quantitative study is needed if these interactions are to be mapped and predictively characterised.

\section{Conclusions and further research}

This research aimed at exploring uncertainty perception as a possible driver for design activity progression with a specific focus on the influence of the dimensions of uncertainty perception on progression with respect to the core actions underpinning design activity: information action, knowledge sharing action, and representation action. Specifically, the following two insights can be derived from the presented work. First, this research showed that dynamic changes in level and decomposed dimensions of uncertainty perception are connected to within and across action design activity progression linking the three core actions (information, knowledge sharing, and representation). Specifically large changes in level of uncertainty perception drive across action activity progression, for example, from information to knowledge sharing, while moderate changes in level drive within action activity progression. Second, this research showed that variations in the decomposed dimensions of uncertainty perception are critically related to across action design activity progression and are linked to a number of characteristic features of design activity. These insights and the conceptualisation of the generic interaction between changing uncertainty perception (as aggregate level and decomposed dimensions) and design activity progression (comprising the three core actions) confirms and elaborates the link between uncertainty perception and design activity. In particular, this research highlights the potential for explaining the interaction between uncertainty perception and design activity progression based on a decomposed model of uncertainty perception.

The main contribution of this research to the design literature lies in the empirical evidence provided for the potential causal mechanism of uncertainty perception in driving design activity progression. Specifically, three implications can be identified. First, this research shows uncertainty perception as a driver for across action design activity progression connecting the core actions - information, knowledge sharing, and representation. This extends prior studies of uncertainty perception, which have focused on individual core actions and sub-types thereof. Second, this research shows that dynamic changes in uncertainty perception in terms of increase and decrease of aggregate uncertainty level are connected to shifts in across action activity progression. Further, the results highlight characteristic patterns in decomposed uncertainty perception associated with such progressions. This extends prior studies, which have focused on binary existence scales of uncertainty perception associated with within action activity progression. Third, the analysis of decomposed uncertainty perception points to an understanding where across action design activity progression can be deconstructed and potentially modelled with respect to different dimensions of uncertainty perception. 
Specifically, this research illustrates distinct patterns of interaction between different dimensions of decomposed uncertainty perception and across action activity progression. This extends prior studies, which have not previously decomposed uncertainty perception or its subsequently varied impact on design activity. Together these implications point to the potential for more elaborate theory building in the uncertainty perception and design activity domain based on bringing together insights from across literature.

This research further has important implications for design practice and education. Specifically, the fundamental role of uncertainty perception in driving design activity suggested by this research gives the following two implications. First, this work provides the first steps towards designers being able to develop observation and management strategies based on uncertainty perception. Such strategies, might allow designers to dynamically monitor uncertainty perception in a given situation and thus more consciously adapt strategies for dealing with it, directed towards increasing the likelihood of successful outcomes. Here design education plays a key role in enabling designers to recognise uncertainty perception as a key influence of their work and providing them with tools to manage it throughout the design process. Second, the decomposed dimensions of uncertainty perception provide a platform for designers to understand the important role implicit and explicit processes play in shaping their work, potentially pointing them towards methods that embrace this dual understanding of cognition. This has particular significance for how designers select and deploy methods and provides a framework for directing discussions of method appropriateness based on awareness of uncertainty perception in a given situation. This again poses a significant challenge for design education by showing the need to educate designers in identifying the points in time of design activity where changes in uncertainty perception associated with both systems of thought occur and how they shape activity progression.

A number of questions for further research are raised. First, the sample size and nature of the current study precludes quantitative generalisation of the results. Rather these findings point to specific research questions: how does the interaction between uncertainty perception and activity progression develop over the course of the design process; and, can activity progression be predicted based on a decomposed model of uncertainty perception? Second, the decomposition of uncertainty perception currently only considers dimensions associated with the systems of thought. Although these provide substantive insights, the management literature makes a number of more specific domainrelated decompositions, and asserts that these also influence activity in distinct ways. As such, further study is needed to determine the relevant components of uncertainty perception in design, and how the varying composition of these affects activity progression. Finally, the dynamic nature of the interaction between uncertainty perception and design activity, and the potential issue of 
endogeneity associated with the measurement of these phenomena points to the need for further methodological research on how such interactions might be investigated. In particular, recent developments in network analysis and signal-processing techniques applied to human behaviour may be relevant here. However, it is clear that protocol analyses alone are limiting in scope and explanatory power given the questions outlined here.

\section{Acknowledgement}

The authors would like to thank the associate editor and reviewers for providing detailed and constructive input helping to develop the final version of this article.

\section{References}

Alexiou, K., Zamenopoulos, T., Johnson, J. H., \& Gilbert, S. J. (2009). Exploring the neurological basis of design cognition using brain imaging: Some preliminary results. Design Studies, 30(6), 623-647.

Anderson, P. (1999). Complexity theory and organization science. Organizational Science, 10(3), 216-232.

Anderson, R. S., Holmes, B., \& Good, R. A. (1973). In vitro bacteridical capacity of Blaberus craniifer hemocytes. Journal of Invertebrate Pathology, 22(1), $127-135$.

Arad, A., \& Gayer, G. (2012). Imprecise data sets as a source of ambiguity: A model and experimental evidence. Management Science, 58(1), 188-202.

Bales, R. F. (2008). The equilibrium problems in small groups. In M. Argyle (Ed.), Social Encounters: Contributions to social interaction (pp. 221-236). Washington, DC, USA: Library of Congress.

Ball, L. J., \& Christensen, B. T. (2009). Analogical reasoning and mental simulation in design: Two strategies linked to uncertainty resolution. Design Studies, 30(2), 169-186.

Ball, L. J., St.B.T. Evans, J., Dennis, I., \& Ormerod, T. C. (1997). Problem-solving strategies and expertise in engineering design. Thinking \& Reasoning, 3(4), 247-270.

Ball, L. J., Onarheim, B., \& Christensen, B. T. (2010). Design requirements, epistemic uncertainty and solution development strategies in software design. Design Studies, 31(6), 567-589.

Bar-Anan, Y., Wilson, T. D., \& Gilbert, D. T. (2009). The feeling of uncertainty intensifies affective reactions. Emotion (Washington, D.C.), 9(1), 123-127.

Bedford, T., \& Cooke, R. (2001). What is Uncertainty? In T. Bedford, \& R. Cooke (Eds.), Probabilistic risk analysis: Foundations and methods (pp. 17-38) Cambridge, UK: Cambridge University Press.

Bedny, G. Z., \& Karwowski, W. (2004). Activity theory as a basis for the study of work. Ergonomics, 47(2), 134-153.

Belkin, N. J., Oddy, R. N., \& Brooks, H. M. (1982). ASK for information retrieval: Part I. Background and theory. Journal of Documentation, 38(2), $61-71$.

Berchicci, L., \& Tucci, C. L. (2010). There is more to market learning than gathering good Information: The role of shared team values in radical product definition*. Journal of Product Innovation Management, 27(7), 972-990.

Beresnev, A. V., Shestirko, L. I., \& Nazarenko, P. M. (1983). Surgical method of treatment of liver cirrhosis. Klinicheskaia Khirurgiia, 21(9), 43-45. 
Berger, C. R., Gudykunst, W. B., \& Deryin, B. (1991). Uncertainty and communication. Basingstoke, UK: Palgrave Macmillan 21-66.

Beynon, M. J., Curry, B., \& Morgan, P. H. (2000). The dempster-shafer theory of evidence: An alternative approach to multicriteria decision modelling. Omega: International Journal of Management Science, 28(1), 37-50.

Bilda, Z., \& Gero, J. S. (2007). The impact of working memory limitations on the design process during conceptualization. Design Studies, 28(4), 343-367.

Cagan, J., Dinar, M., Shah, J. J., Leifer, L., Linsey, J., Smith, S. M., et al. (2013). Empirical studies of design thinking: Past, present, future. In DETC '13: ASME 2013 design engineering technical conferences (pp. 1-12). Portland: Oregon, USA.

Cash, P., Hicks, B. J., \& Culley, S. J. (2013). A comparison of designer activity using core design situations in the laboratory and practice. Design Studies, 34(5), 575-611.

Cash, P., Hicks, B., \& Culley, S. (2015). Activity theory as a means for multi-scale analysis of the engineering design process: A protocol study of design in practice. Design Studies, 38(May), 1-32.

Cash, P., \& Kreye, M. E. (2017). Uncertainty Driven Action (UDA) model: A foundation for unifying perspectives on design activity. Design Science. in press.

Cash, P., \& Maier, A. (2016). Prototyping with your hands: The many roles of gesture in the communication of design concepts. Journal of Engineering Design, 27(1-3), 118-145.

Chiu, C.-M., Hsu, M.-H., \& Wang, E. T. G. (2006). Understanding knowledge sharing in virtual communities: An integration of social capital and social cognitive theories. Decision Support Systems, 42(3), 1872-1888.

Christensen, B. T., \& Ball, L. J. (2016). Creative analogy use in a heterogeneous design team: The pervasive role of background domain knowledge. Design Studies, 46, 38-58.

Christensen, B. T., \& Ball, L. J. (2017). Fluctuating epistemic uncertainty in a design team as a metacognitive driver for creative cognitive processes. In B. T. Christensen, L. J. Ball, \& K. Halskov (Eds.), Analysing design Thinking: Studies of cross-cultural Co-Creation (pp. 249-270). CRC Press.

Cross, N. (2007). Forty years of design research. Design Studies, 28(1), 1-4.

Daft, R. L., \& Lengel, R. H. (1983). information richness: A new approach to managerial behavior and organization design. In B. Staw, \& L. L. Cummings (Eds.), Research in Organizational behavior. Connecticut: JAI Press.

Daft, R. L., \& Lengel, R. H. (1986). Organizational information requirements, media richness and structural design. Management Science, 32(5), 554-571.

De Brentani, U., \& Reid, S. E. (2012). The fuzzy front-end of discontinuous innovation: Insights for research and management. Journal of Product Innovation Management, 29(1), 70-87.

DeFranco, J. F., Neill, C. J., \& Clariana, R. B. (2011). A cognitive collaborative model to improve performance in engineering teams - a study of team outcomes and mental model sharing. Systems Engineering, 14(3), 305-326.

Deken, F., Kleinsmann, M., Aurisicchio, M., Lauche, K., \& Bracewell, R. (2012). Tapping into past design experiences: Knowledge sharing and creation during novice-expert design consultations. Research in Engineering Design, 23(3), $203-218$.

Dorst, K. (1995). Analysing design activity: New directions in protocol analysis. Design Studies, 16(2), 139-142.

Dorst, K., \& Cross, N. (2001). Creativity in the design process: Co-evolution of problem-solution. Design Studies, 22(5), 425-437. 
Downey, H. K., \& Slocum, J. W. (1975). Uncertainty: Measures, research, and sources of variation. Academy of Management Journal, 18(3), 562-578.

Ellsberg, D. (2001). Risk, ambiguity, and decision. New York, NY, USA: Routledge - Studies in Psychology.

Fiske, S. T., \& Taylor, S. E. (2008). Attention and encoding. In Social cognition. From Brains to culture (pp. 51-74). Sage Publications Inc.

Galbraith, J. R. (1974, May). Organization Design: An information processing view. Interfaces.

Gerber, E., \& Carroll, M. (2012). The psychological experience of prototyping. Design Studies, 33(1), 64-84.

Goh, Y. M., Newnes, L. B., Mileham, A. R., Mcmahon, C. A., \& Saravi, M. E. (2010). Uncertainty in through-life costing - review and perspectives. IEEE Transactions on Engineering Management, 57(4), 689-701.

Gonçalves, M., Cardoso, C., \& Badke-Schaub, P. (2016). Inspiration choices that matter: The selection of external stimuli during ideation. Design Science, 2(November), e10.

Goodwin, P., \& Wright, G. (1993). Improving judgmental time series forecasting: A review of the guidance provided by research. International Journal of Forecasting, 9(2), 147-161.

Gursoy, B., \& Ozkar, M. (2015). Visualizing making: Shapes, materials, and actions. Design Studies, 41, 29-50.

Hand, D. J., \& Walley, P. (1993). Statistical reasoning with imprecise probabilities. Applied statistics. London, UK: Chapman and Hall.

Harvey, N. (2001). Improving judgement in forecasting. In J. S. Armstrong (Ed.), Principles of forecasting: A handbook for researchers and practitioners (pp. 59-80). New York, NY, USA: Springer Science \& Business Media.

Holland, J. H. (1995). Hidden order. Reading, MA, USA: Addison-Wesley.

Hult, G. T. M., Ketchen, D. J., \& Slater, S. F. (2004). Information processing, knowledge development, and strategic supply chain performance. Academy of Management Journal, 47(2), 241-253.

Hurley, R. J., Kosenko, K. A., \& Brashers, D. (2011). Uncertain Terms: Message features of online cancer news. Communication monographs. Routledge.

ISO. (2009). Risk management - principles and guidelines. International Organization for Standardization.

Kim, K., \& Lee, K. (2016). Collaborative product design processes of industrial design and engineering design in consumer product companies. Design Studies, $46,226-260$.

Kleinsmann, M., \& Valkenburg, R. (2008). Barriers and enablers for creating shared understanding in co-design projects. Design Studies, 29(4), 369-386.

Kreye, M. E. (2013). Uncertainty awareness in support service contracts. EurOMA - european operations management association. Dublin: Irleand.

Kreye, M. E. (2017). Relational uncertainty in service dyads. International Journal of Operations \& Production Management, 37(3), 363-381.

Kreye, M. E., Goh, Y. M., \& Newnes, L. B. (2011). Manifestation of uncertainty a classification. In ICED'11-International conference on engineering design. Copenhagen, Denmark.

Kreye, M. E., Goh, Y. M., Newnes, L. B., \& Goodwin, P. (2012). Approaches of displaying information to assist decisions under uncertainty. Omega - International Journal of Management Science, 40(6), 682-692.

Kreye, M. E., Newnes, L. B., \& Goh, Y.-M. (2012). Uncertainty in competitive bidding: A framework for product-service systems. Production Planning \& Control, 25(6), 462-477. 
Krippendorff, K. (1981). Content analysis: An introduction to its methodology. In F. G. Kline, \& S. H. Evans (Eds.), The sage CommText series, Vol. Second. Thousand Oaks, CA, CA, USA: Sage.

Krippendorff, K. (2004). Reliability in content Analysis: Some common misconceptions and recommendations. Human Communication Research, 30(3), 411-433.

Kuutti, K. (1995). Activity Theory as a potential framework for human-computer interaction research. In B. Nardi (Ed.), Context and Consciousness: Activity theory and human computer interaction (pp. 17-44). . Cambridge, US: Cambridge: MIT Press.

Lane, D. A., \& Maxfield, R. R. (2005). Ontological uncertainty and innovation. Journal of Evolutionary Economics, 15(1), 3-50.

Langer, E. J., \& Roth, J. (1975). Heads I win, tails is Chance: The illusion of control is a function of the sequence of outcomes in a purely chance task. Journal of Personality and Social Psychology, 32(1975), 951-955.

Lawrence, M., \& Makridakis, S. (1989). Factors affecting judgmental forecasts and confidence intervals. Organizational Behavior and Human Decision Processes, 43(2), 172-187.

Martinec, T., Skec, S., \& Storga, M. (2017). Exploring the decomposition of team design activity. In ICED 17 international conference on engineering design (pp. 1-10).

McKenna, J. (2002). Book Review: Communicating Uncertainty: Media coverage of new and controversial science. Journalism. Mahwah, NJ, USA: Lawrence Erlbaum Associates.

Moller, B., \& Beer, M. (2008). Engineering computation under uncertainty-Capabilities of non-traditional models. Computers \& Structures, 86(10), 1024-1041.

Morone, A., \& Morone, P. (2008). Guessing games and people Behaviours: What can we learn? Advances in Decision Making Under Risk and Uncertainty 205-217.

Neuendorf, K. A. (2002). The content analysis guidebook. Thousand Oaks, USA: SAGE Publications, Incorporated.

O'Connor, G. C., \& Rice, M. P. (2013). A comprehensive model of uncertainty associated with radical innovation. Journal of Product Innovation Management, 30(SUPPL 1), 2-18, (Journal Article).

Oberkampf, W. L., DeLand, S. M., Rutherford, B. M., Diegert, K. V., \& Alvin, K. F. (2002). Error and uncertainty in modeling and simulation. Reliability Engineering \& System Safety, 75(3), 333-357.

Oppl, S., \& Stary, C. (2013). Facilitating shared understanding of work situations using a tangible tabletop interface. Behaviour \& Information Technology, 33(6), $619-635$.

Robinson, M. A. (2010). Work sampling: Methodological advances and new applications. Human Factors and Ergonomics in Manufacturing \& Service Industries, 20(1), 42-60.

Sanders, E. B. N., \& Stappers, P. J. (2014). Probes, toolkits and prototypes: Three approaches to making in codesigning. CoDesign, 10(1), 5-14.

Scaife, M., \& Rogers, Y. (1996). External cognition: How do graphical representations work? International Journal of Human-Computer Studies, 45(2), $185-213$.

Schön, D. A., \& Wiggins, G. (1992). Kinds of seeing and their functions in designing. Design Studies, 13(2), 135-156.

Scrivener, S. A. R., Ball, L. J., \& Tseng, W. (2000). Uncertainty and sketching behaviour. Design Studies, 21(5), 465-481. 
Shafer, G. (1994). The subjective aspect of probability. In G. Wright, \& P. Ayton (Eds.), Subjective probability. New York, NY, USA: John Wiley \& Sons.

Sicotte, H., \& Bourgault, M. (2008). Dimensions of uncertainty and their moderating effect on new product development project performance. $R \& D$ Management, 38(5), 468-479.

Sim, S. K., \& Duffy, A. H. B. (2003). Towards an ontology of generic engineering design activities. Research in Engineering Design, 14(4), 200-223.

Sloman, S. A. (2002). Two systems of reasoning. In T. Gilovich, D. Griffin, \& D. Kahneman (Eds.), Heuristics and Biases: The psychology of intuitive thought (pp. 397-420). New York, NY, US: Cambridge University Press.

Soanes, C. (1928). The Oxford english dictionary *. Canadian Medical Association Journal, 18(4), 464-465.

Song, M., \& Montoya-Weiss, M. M. (2001). The effect of perceived technological uncertainty on Japanese new product development. Academy of Management Journal, 44(1), 61-80.

Song, M., Van Der Bij, H., \& Weggeman, M. (2005). Determinants of the level of knowledge application: A knowledge-based and information-processing perspective. Journal of Product Innovation Management, 22(5), 430-444.

Stempfle, J., \& Badke-Schaub, P. (2002). Thinking in design teams - an analysis of team communication. Design Studies, 23(5), 473-496.

Storga, M., Andreasen, M. M., Marjanovic, D., Štorga, M., Andreasen, M. M., \& Marjanović, D. (2010). The design ontology: Foundation for the design knowledge exchange and management. Journal of Engineering Design, 21(4), 427-454.

Torgerson, D. J., \& Torgerson, C. J. (2003). Avoiding bias in randomised controlled trials in educational research. British Journal of Educational Studies, 51(1), 36-45.

Tracey, M. W., \& Hutchinson, A. (2016). Uncertainty, reflection, and designer identity development. Design Studies, 42, 86-109.

Tversky, A., \& Kahneman, D. (1974). Judgment under uncertainty: Heuristics and biases. Science, 185(4157), 1124-1131.

Ulrich, K. T., \& Eppinger, S. D. (2003). Product design and development, Vol. 5th. New York, USA: MvGraw-Hill.

Van Der Lugt, R. (2005). How sketching can affect the idea generation process in design group meetings. Design Studies, 26(2), 101-112.

Walker, W. E., Harremoes, P., Rotmans, J., Van der Sluijs, J. P., Van Asselt, M. B. A., Janssen, P., et al. (2003). Defining uncertainty: A conceptual basis for uncertainty management in model based decision support. Integrated Assessment, 4(1), 5-17.

Wasiak, J., Hicks, B. J., Newnes, L., Dong, A., \& Burrow, L. (2010). Understanding engineering email: The development of a taxonomy for identifying and classifying engineering work. Research in Engineering Design, 21(1), 43-64.

Wasiak, J., Hicks, B., Newnes, L., Loftus, C., Dong, A., \& Burrow, L. (2011). Managing by E-mail: What e-mail can do for engineering project management. IEEE Transactions on Engineering Management, 58(3), 445-456.

Weber, R. P. (1990). Basic content analysis. In M. S. Lewis-Beck (Ed.), Journal of the American Statistical association (2nd ed.)., Vol. 82 Newbury Park, CA, USA: Sage Publications, Inc.

Wiltschnig, S., Christensen, B. T., \& Ball, L. J. (2013). Collaborative problemsolution co-evolution in creative design. Design Studies, 34(5), 515-542.

Windschitl, P. D., \& Wells, G. L. (1996). Measuring psychological uncertainty: Verbal versus numeric methods. Journal of Experimental Psychology: Applied, 2(4), 343-364, US: American Psychological Association). 
Wynn, D. C., Grebici, K., \& Clarkson, P. J. (2011). Modelling the evolution of uncertainty levels during design. International Journal on Interactive Design and Manufacturing, 5(3), 187-202.

Xenakis, I., \& Arnellos, A. (2013). The relation between interaction aesthetics and affordances. Design Studies, 34(1), 57-73.

Yu, B. Y., Honda, T., Sharqawy, M., \& Yang, M. (2016). Human behavior and domain knowledge in parameter design of complex systems. Design Studies $1-26$.

Zimmermann, H. J. (2000). Application-oriented view of modeling uncertainty. European Journal of Operational Research, 122(2), 190-198. 